\title{
Erratum to: Investigating a safe ventilation rate for the prevention of indoor SARS transmission: An attempt based on a simulation approach
}

\author{
Yi Jiang $(\bowtie)$, Bin Zhao, Xiaofeng Li, Xudong Yang, Zhiqin Zhang, Yufeng Zhang \\ Department of Building Science, School of Architecture, Tsinghua University, Beijing 100084, China
}

๑ Tsinghua University Press and Springer-Verlag Berlin Heidelberg 2009

\section{Erratum to}

BUILD SIMUL (2009) 2: 281-289

DOI 10.1007/s12273-009-9325-7

In the online version of the original article, the description of Eq. (2) on page 283 contains an error. Instead of

$F_{j, i}=f\left(P_{j}-P_{i}\right)$

where $f$ is the coefficient for the calculating airflow rate, and $P_{j}-P_{i}$ is the pressure drop along the flow path.

It should read

$F_{j, i}=f\left(P_{j}-P_{i}\right)$

where $f$ represents the function correlating the pressure drop with the airflow rate, and $P_{j}-P_{i}$ is the pressure drop along the flow path. 